\title{
La discriminación vive en las calles. Análisis de vivencias rutinarias que configuran discriminación contra las mujeres ${ }^{*}$
}

\author{
Discrimination Lives on the Streets. Analysis of Routine \\ Experiences that Constitute Discrimination against Women
A discriminação vive nas ruas. Análise de experiências cotidianas que constituem discriminação contra a mulher

\author{
Lina Milena García Sierra ${ }^{\star *}$
}

FeCha de ReCepción: 29 de nOVIEMbre de 2020. FeCha de AProbación: 8 DE JUlio de 2021

Doi: https://doi.org/10.12804/revistas.urosario.edu.co/sociojuridicos/a.10009

Para citar este artículo: García Sierra, L. M. (2022). La discriminación vive en las calles. Análisis de vivencias rutinarias que configuran discriminación contra las mujeres. Estudios Socio-Jurídicos, 24(1), 299-326. [Publicación electrónica previa a la impresión] https://doi.org/10.12804/revistas.urosario.edu.co/sociojuridicos/a.10009

\section{RESUMEN}

El presente artículo hace un recorrido por la construcción del discurso, sus alcances en la construcción de realidades y de representación de grupos identitarios, ello con el fin de llevar al lector a un análisis profundo sobre la discriminación como categoría social y jurídico-penal. Se usaron como insumo de investigación sesiones de charlas adelantadas con mujeres sobre las violencias contra las mujeres, el sexismo, el discurso de odio y la configuración de agencias tanto individuales como grupales.

Finalmente, se aborda la eficiencia y coherencia entre el marco normativo colombiano en materia de prevención de violencias contra la mujer y el impacto en la reducción de delitos contra las mujeres por razones de género. Se propone a su vez la intervención desde la política criminal para que desde un enfoque de género se logren estrategias eficaces para mejorar las condiciones de vida de las mujeres.

Palabras clave: discriminación; violencia de género; discurso.

* El artículo no ha sido presentado en ningún congreso ni ponencia.

** Abogada de la Universidad Santo Tomás; magíster en Estudios Culturales de la Universidad de los Andes; doctoranda en Derecho de la Universidad Nacional de Colombia. Docente de la Maestría en Derecho Penal de la Universidad Santo Tomás. Correo electrónico institucional: linagarcia@usantotomas.edu.co. ORCID: https://orcid.org/0000-0002-0531-4880 


\section{ABSTRACT}

This article takes a tour of the construction of discourse and its influence on the construction of realities and on the representation policies of identity groups. It leads the reader to a deep analysis of discrimination as a legal, social, and criminal category. The methodology included digital sessions with women discussing violence against women, sexism, hate speech, and the configuration of both individual and group agencies.

Finally, the article addresses the efficiency and coherence between the Colombian normative framework regarding the prevention of violence against women and the impact on reducing crimes against women based on gender. As a result, we proposed effective strategies to achieve a gender perspective that improves the living conditions of women through a comprehensive intervention in criminal policy.

Keywords: Discrimination; gender violence; discourse.

\section{RESUMO}

Este artigo faz um percurso pela construção do discurso, seu alcance na construção de realidades e na representação de grupos identitários, a fim de levar o leitor a uma análise profunda da discriminação como categoria social e jurídico penal. Sessões de bate-papo realizadas com mulheres sobre violência contra mulheres, sexismo, discurso de ódio e a configuração de agências individuais e grupais, foram usadas como dados de pesquisa.

Por fim, aborda-se a eficiência e a coerência entre o marco regulatório colombiano em relação à prevenção da violência contra a mulher e o impacto na redução de crimes contra as mulheres por motivos de gênero. Por sua vez, propõe-se a intervenção da política criminal para que sejam alcançadas estratégias efetivas desde uma perspectiva de gênero para melhoria das condições de vida das mulheres.

Palavras-chave: discriminação; violência de gênero; discurso. 
El discurso construye realidades y opera en cada espacio de la vida rutinaria, permite la definición de categorías, la creación de significado y la determinación de las características de referencia de cada grupo identitario. El discurso más allá de las expresiones orales lingüísticas abarca también los textos, gestos, cuerpos y, en general, las prácticas culturales que dan sentido a las realidades.

El discurso es una fuente de poder, lo crea, lo consolida y lo destruye. Para Foucault, se debe tener en cuenta el impacto que tiene en la creación de realidades; en $E l$ orden del discurso (1992), describe su preocupación por lo inmanejable de esta práctica, pues requiere de una cuidadosa revisión sobre las posibilidades que va creando a su camino. El discurso determina qué es verdad y qué no lo es, por ello tiene también la posibilidad de etiquetar a los individuos entre quienes cumplen con sus postulados de validez y quienes quedarán reseñados como lo otro, llevando consigo todas las consecuencias en la distribución de derecho que eso implica para un grupo u otro.

Austin (2014) ya advertía que se pueden hacer cosas con las palabras y en el mundo contemporáneo podemos afirmar que nada es tan tangible como el discurso de odio. La hostilidad que se deriva de procesos en que se hiere con las palabras determina los sujetos subalternos en las sociedades. Bajo las políticas de representación que ha impuesto la modernidad se da sentido a la otredad a partir de la división binaria de los sujetos, es decir, somos (reafirmamos nuestra identidad) en la medida que no cumplimos ciertos patrones de características rechazables, "anormales" o patológicas.

El desarrollo de la sociedad para configurar las relaciones de poder surge entonces de la construcción colectiva de imaginarios que determinan cómo actuar, interactuar y, en general, performar las identidades; de tal manera que como individuos nos validamos en rituales de verdad (Foucault, 2010) que nos acercan al ideal del deber ser.

En contraposición de la medida de validez, se determinan las características poco deseables que configurarán al Otro, ellas son asociadas a factores de subordinación, diversidad, no homogeneidad, anormalidad y las demás figuras de alerta que etiquetan a un sujeto o grupo como lejano, peligroso, indeseable, etc. 
Los grupos históricamente vulnerados han estado sujetos a esta dualidad que supone el discurso en la representación de identidades; por ello, población judía, LGBTIQ, negritudes, indígenas, mujeres y otros han sido constantemente deshumanizados y sometidos a tratos desiguales de forma sistemática.

Tanto Austin (2014) como Butler (2004) indican que la discriminación contra grupos etiquetados como subalternos se origina en los actos de habla ilocucionarios, es decir, los que al expresarse realizan en sí mismos la acción que enuncian. La discriminación se ejecuta en el momento en que se da un trato distinto a un sujeto por la pertenencia a un grupo; Chimamanda Adichie lo explica bajo la siguiente fórmula: "[...] si criticas $\mathrm{X}$ en las mujeres pero no lo criticas en los hombres, tal vez no tengas un problema con $\mathrm{X}$, sino con las mujeres. Sustituye la $\mathrm{X}$ por palabras como ira, ambición, brusquedad, tozudez, frialdad, crueldad" (2018, pp. 45, 46).

Si bien la discriminación no cumple su ciclo absoluto en el acto de habla, sí puede manifestarse y cumplir su objetivo en determinados actos de habla, por ejemplo, Butler (2004) usa el caso estadounidense R. A. V. v. St. Paul, en el cual un joven blanco prende una cruz en llamas y la sostiene en la acera frente a la casa de una familia negra en Estados Unidos, aquí el acto mismo envía un mensaje de rechazo a la presencia de la familia en la zona, recuerda y actualiza los postulados del Ku Klux Klan y a la vez logra instaurar en la familia un estado de temor, sentimiento de fragilidad ante la amenaza y la interiorización del rechazo externo hacia ellos.

En el caso relatado por Butler en Lenguaje, poder e identidad (2004), la discriminación racial no se origina ni termina con el acto del joven blanco, sin embargo, esa situación en específico sí recoge todo el proceso de creación de significado racista y lo expresa en un solo momento, logrando allí mismo la recepción del mensaje y la percepción de discriminación por parte de las víctimas de esta.

La discriminación es un largo camino que va engranando situaciones adversas para los grupos o individuos contra quienes se dirige, iniciando en expresiones de habla y avanzando incluso hasta actos de violencia física, para el caso de las mujeres, como el feminicidio. 
Esta situación de relación estrecha entre actos de discriminación y su materialización en la violencia basada en género fue definida por la Cedaw así:

Artículo $1^{\circ}$. [...] la expresión "discriminación contra la mujer" denotará toda distinción, exclusión o restricción basada en el sexo que tenga por objeto o resultado menoscabar o anular el reconocimiento, goce o ejercicio por la mujer, independientemente de su estado civil, sobre la base de la igualdad del hombre y la mujer, de los derechos humanos y las libertades fundamentales en las esferas política, económica, social, cultural y civil o en cualquier otra esfera (Naciones Unidas, 1979).

La evolución del concepto de discriminación contra las mujeres, en el entendido de incluir allí también todo acto de violencia, al revelar que en ellos se expresa la subordinación impuesta a las mujeres y la consolidación de un sistema patriarcal que minimiza el rol de las mujeres en la sociedad, ha sido relatado y visibilizado por la Corte Interamericana de Derechos Humanos, además de advertir sobre la participación del Estado en estos actos de discriminación al actuar de forma ineficiente, tardía u omitir la debida diligencia:

La Corte estima que la violencia basada en el género, es decir la violencia dirigida contra una mujer por ser mujer o la violencia que afecta a la mujer de manera desproporcionada, es una forma de discriminación en contra de la mujer [...].

La Corte reitera que la ineficacia judicial frente a casos individuales de violencia contra las mujeres propicia un ambiente de impunidad que facilita y promueve la repetición de los hechos de violencia en general y envía un mensaje según el cual la violencia contra las mujeres puede ser tolerada y aceptada, lo que favorece su perpetuación y la aceptación social del fenómeno, el sentimiento y la sensación de inseguridad de las mujeres, así como una persistente desconfianza de estas en el sistema de administración de justicia. Dicha ineficacia o indiferencia constituye en sí misma una discriminación [de la mujer] en el acceso a la justicia. Por ello, cuando existan indicios o sospechas 
concretas de violencia de género, la falta de investigación por parte de las autoridades de los posibles móviles discriminatorios que tuvo un acto de violencia contra la mujer, puede constituir en sí misma una forma de discriminación basada en el género (Veliz Franco y otros vs. Guatemala, 2014).

La discriminación contra las mujeres se gesta desde actos rutinarios que, si bien no están incluidos en el marco de interés del derecho penal, sí constituyen el contexto que debe analizarse e incluirse como variable en los procesos penales por delitos relacionados con la violencia basada en género ( $\mathrm{VBG}$ ).

En el mismo sentido debe tenerse en cuenta por parte del Estado, al momento de plantear la política criminal, que de forma integral y transversal debe garantizar a su vez acciones de prevención, visibilización de las violencias y acceso a la justicia que permita superar la obstrucción histórica a este derecho fundamental para los grupos históricamente vulnerados, en este caso las mujeres.

Este texto, que se suscribirá en la tipología de artículo de reflexión, responde a un problema de investigación que surge de la inoperancia del derecho penal para desincentivar la ocurrencia de delitos de VBG contra las mujeres, la nula capacidad que ha logrado construir este para exhortar al Estado en el reconocimiento de su responsabilidad en estos escenarios y la omisión en vincular planes de acción posteriores a los fallos que logren intervenciones estructurales de prevención a la VGB.

A su vez, el presente artículo se escribe con el fin de dar unas bases de análisis que permitan entrelazar las realidades territoriales de las mujeres con los objetivos del derecho penal y sentar una crítica que aporte a la exigencia de una política criminal que se establezca de forma transversal, interdisciplinar y plurivocal, logrando un enfoque de género en toda su creación e implementación.

En el desarrollo del presente texto realizaremos un acercamiento a las formas en que un grupo de mujeres de Usaquén han vivido el sexismo en sus mundos inmediatos, las implicaciones posteriores de tales actos discriminatorios y las visiones propias de ellas sobre las instituciones tanto sociales como públicas en relación con la ocurrencia de estos hechos. 
Estas mujeres conformaban un grupo de trece personas aproximadamente (aunque variaba la asistencia en cada sesión), de estrato socioeconómico 1 o 2 en el noroccidente de Bogotá, sector El Codito de la localidad de Usaquén. Todas ellas son madres cabeza de familia resultado de embarazos adolescentes mayoritariamente, que oscilan entre los 16 y 25 años, con una excepción de una mujer mayor de 40 años.

Todas asisten a un programa de jardín comunitario para sus hijos o hijas, en donde reciben formación corta sobre distintos temas, servicios relacionados con el cuidado de los y las menores y una ayuda de mercado mensual para sus familias. El trabajo de campo se desarrolló con sesiones semanales de acuerdo con un programa en el que discutíamos sobre nuestras historias de vida; las violencias de género; el disciplinamiento de los cuerpos; el discurso sexista y prácticas culturales que las llevaron al reconocimiento propio como sujeto femenino individual y como agente colectivo.

En un segundo momento analizaremos el alcance del sexismo en la ocurrencia de delitos relacionados con la violencia de género y el actuar eficaz o no del derecho penal sobre esta materia en Colombia.

Finalmente, propondremos una intervención amplia por parte del Estado para que a partir de la política criminal se realicen actos de prevención de la VBG y se ponga especial atención sobre las etapas que tienen una afectación menor a los bienes jurídicos pero que son las que inician el proceso de consolidación, actualización y reproducción del sexismo en el día a día.

\section{Hablemos con las mujeres sobre sexismo}

Durante cuatro sesiones dialogamos ${ }^{1}$ con un grupo mujeres que habitan en Usaquén, localidad de Bogotá, todas ellas vinculadas a un espacio del Instituto Colombiano de Bienestar Familiar que se denomina centros de desarrollo integral (CDI), como madres de niños y niñas de 2 a 5 años que reciben "atención integral y diferencial, a través de acciones

1 Las sesiones se efectuaron durante los meses de octubre y noviembre del año 2020, de forma virtual a través de la plataforma Meet. 
pedagógicas, de cuidado calificado y nutrición" (ICBF, 2020); además del servicio a los y las menores, los CDI realizan talleres de prevención de embarazos subsecuentes, formación en derechos de gestantes, lactantes y prevención de violencias, así como un acompañamiento constante a las madres en sus procesos de acceso a la salud y otros derechos. En el transcurso de las sesiones se desarrollaron cuatro módulos que abarcaron temas de violencias, construcción de identidad, discurso sexista y el encuentro en la agencia individual y colectiva; también intercambiaron experiencias y permitieron reflexiones sobre los procesos de construcción de las violencias contra las mujeres.

Las sesiones se guiaron bajo el objetivo de "construir colectivamente reflexiones sobre las historias de vida de las mujeres, las violencias que vivimos en entornos públicos y privados e indagar sobre la construcción de agencias individuales y como sector".

\section{Saberse mujer}

En la primera sesión se planteó la pregunta de ¿en qué momento se dan cuenta que son mujeres?, buscando conocer el punto de inicio en el que se reconocen a sí mismas como parte de un grupo con unas características específicas, y cómo ocurre ese primer momento en cada una de sus historias. Las respuestas fueron diversas.

Una parte del grupo habló de situaciones en las que el disciplinamiento del cuerpo daba pauta para que interiorizaran su pertenencia a un sector de la población, el de las mujeres, anécdotas relacionadas con juegos 'masculinos' con primos, hermanos y amigos que fueron prohibidos por los padres al considerar que no eran conductas en que ellas como mujeres debieran participar; instrucciones sobre cómo performar la feminidad, por ejemplo, en la forma adecuada de sentarse (cerrar siempre las piernas), vestirse (a partir de determinada edad comenzar a usar vestidos), mostrarse ante la sociedad (no puede ser un 'marimacho').

Algunas mujeres mencionaron que la distribución de las tareas en el hogar las hacía saber desde pequeñas que eran mujeres. Acá los relatos se basan en la destinación de actividades de cuidado y trabajo doméstico que solo recaían sobre ellas, sin importar si eran mayores o menores que sus hermanos hombres; así, labores de cocina, servir 
comida, lavar platos, alistar ropa, cuidar de los más pequeños o cualquier tarea del hogar estaba predeterminada para la ejecución por parte de las mujeres de la casa.

Diferentes intervenciones de la primera sesión recordaron la primera menstruación como el momento de darse cuenta de su feminidad, no solo por la sangre y la explicación de sus madres sobre el significado de ella (dejar de ser una niña), sino por el inicio de la pubertad y la forma de relacionarse con el sexo masculino a partir de allí; mencionaban algunas de las mujeres participantes que comenzaban a sentirse atraídas por los hombres, así como a saberse objeto de deseo del sexo opuesto.

Algunas de las participantes coincidieron en que se dan cuenta de su rol y de la pertenencia al grupo 'mujeres' al momento de quedar embarazadas y afrontar un parto; algunas porque el anuncio del embarazo las deja frente a un ejercicio de maternidad individual al no contar con el apoyo de sus parejas, quienes deciden desaparecer, cuestionar la paternidad o pedirles que aborten sin que ese sea el deseo de ellas, descubriendo allí que la sociedad, familia y entorno inmediato les da a entender que deben iniciar su 'rol natural' de madres, etapa que ellas manifiestan asumir con fuerza y con convicción de poder: "Sacar adelante a sus hijos solas".

Otras mencionan que la maternidad es el momento de click con su identidad de mujeres, al verse enfrentadas a los cambios hormonales, al parto violento en donde las gritan, les dan órdenes y deciden sobre ellas, pero también en la etapa de lactancia, cuando tienen muchas dudas, incluso depresión, y la atención se centra sobre los bebés mas no en las madres, allí las recoge un sentimiento de fragilidad, soledad e invisibilización, pues la importancia recae en la maternidad y no en ellas como mujeres.

Finalmente, algunas de ellas mencionan que el momento en que se dan cuenta que son mujeres es cuando deben cambiar su forma de vestir para evitar los comentarios inapropiados e incómodos en la calle sobre sus cuerpos, cuando deciden que deben usar ropa ancha para que sus cuerpos no estén sometidos a una opinión pública que no han solicitado.

Se evidencia la diversidad en la respuesta de las mujeres sobre su momento y forma de relacionarse con su yo femenino, del momento 
de descubrirse mujeres socialmente, sin embargo, hay identidad en las respuestas al identificar que es siempre un tercero individualizable o abstracto quien las hace notar su particularidad de mujeres, ya sea por las expectativas que se crean sobre ellas ante algunas etapas de sus vidas, como el momento en que menstrúan por primera vez o cuando deciden ser madres, o ante sus formas de vivir el mundo, ya sea en el hogar, escenarios de juegos cuando niñas o interacciones con el espacio público que las afrontan al acoso callejero.

Las mujeres nos sabemos mujeres en la medida que no representamos lo masculino, en que se nos prohíben ciertas acciones, se nos alerta de la vulnerabilidad o el peligro al que estamos sometidas por las 'realidades' del mundo exterior o porque al desviarnos del deber ser de la feminidad se nos envían señales que nos redireccionan para mantenernos en el rol y los estándares supuestos.

El discurso que determina la discriminación y en este caso el sexismo, que se manifiesta, como lo menciona Marcela Lagarde, basándose en el androcentrismo: "La mentalidad androcéntrica permite considerar valorativamente y apoyar socialmente que los hombres y lo masculino son superiores, mejores, más adecuados, más capaces y más útiles que las mujeres" (1996, p. 79).

Así se logra interpelar a las mujeres para que construyan su identidad desde una edad muy temprana bajo los estereotipos de género, sin procesos reflexivos ni críticos al momento en que enfrentan tales situaciones. El papel de la sociedad es repetir y validar que tal distribución o etiqueta sobre lo que debe ser femenino y lo que no se cumple a cabalidad.

Veremos más adelante que, no obstante este proceso parece contar con un ciclo perfecto, las vivencias posteriores de las mujeres y sus propios cuestionamientos en momentos distintos frente a los hechos que dan inicio a su relación con la feminidad o el saberse mujeres producen efectos de resignificación en el discurso, reconocimiento de violencias y desacreditación de los estereotipos de género para visibilizar los puntos fallidos de sus propios procesos de identidad, así como generar la traza de estrategias que permitan ser críticas frente a nuevos escenarios estereotipados que afrontan en su día a día, e incluso romper ciclos de aprendizajes binarios en la crianza de sus hijos e hijas. 


\section{Sexismo en el día a día}

En una segunda sesión pusimos en debate el lenguaje sexista y su presencia en la cotidianidad. Allí las mujeres participantes en las sesiones expusieron que el sexismo, según la definición de trato desigual que se refirió en el módulo, ${ }^{2}$ se manifestaba para ellas en la ponderación constante de sus cuerpos, es decir, en la valoración de aprobación o desaprobación según los comentarios masculinos y de la sociedad, y a la vez en la imposición de cursos a sus proyectos de vida, identidades y formas de participar en el mundo.

Una de las mujeres mencionó que prefería no tener redes sociales para no someterse a los comentarios no solicitados con que se encontraba de forma frecuente; otra de ellas menciona que, si bien esta situación también le es familiar, ella optó por no darle importancia y no responder a los malos comentarios que pudieran hacerle. Las redes sociales son lugares virtuales por excelencia hostiles, pues la posibilidad de actuar bajo el anonimato permite que las personas emitan mensajes de odio sin temer por las consecuencias (Kohn, 2018).

Por otra parte, una de las mujeres comentó que había vivido el sexismo cuando en el colegio un profesor se refería a ella como la 'gorda gafufa', mostrando rechazo por su apariencia y además deslegitimando su trabajo académico por características físicas que el docente consideraba molestas en una niña. También mencionó que había sido discriminada cuando practicaba fútbol con los niños y le recordaban constantemente que no era un lugar para ella.

Para concluir esta sesión, una de las participantes relata que al haber elegido como profesión la zootecnia se veía enfrentada a entornos sexistas de forma permanente: primero, porque era una carrera que no se consideraba propia de las mujeres; segundo, porque las ofertas laborales mayoritariamente limitaban la postulación de hojas de vida a hombres; y tercero, porque se creía en el gremio que una mujer no

2 Sexismo: "Conjunto de todos y cada uno de los métodos empleados en el seno del patriarcado para poder mantener en situación de inferioridad, subordinación y explotación al sexo dominado: el femenino. El sexismo abarca todos los ámbitos de la vida y las relaciones humanas, de modo que es imposible hacer una relación, no exhaustiva, sino ni tan siquiera aproximada de sus formas de expresión y puntos de incidencia, [...]" (Sau, 2000, p. 257). 
podía realizar actividades de finca, entonces quedaba relegado su ejercicio profesional a especies de menor tamaño.

El sexismo como acto de trato subordinado a las mujeres, ya sea en la limitación a su personalidad o desarrollo de actividades rutinarias generada por comportamientos patriarcales, se hace palpable en todas las historias de vida, ya sea por actos directos en los que les cuestionan sus cuerpos, decisiones, profesiones o actuar, o en situaciones en las que se les priva de oportunidades por su género. Todas ellas reconocían que las situaciones relatadas constituían momentos de discriminación como manifestación del sexismo sobre los que no estaban de acuerdo $\mathrm{y}$, en general, generaban rabia, inconformismo y frustración, pues obstaculiza su desarrollo personal, social y hasta profesional, cumpliendo una característica central del sexismo o de cualquier discurso de odio, la deshumanización del sujeto o grupo receptor del odio (Tsesis, 2002).

\section{Violencias basadas en género. De la ley a la vida de las mujeres}

En una tercera sesión dialogamos sobre la violencia contra las mujeres, allí inicialmente se realizó una explicación de los cinco tipos de violencias contra las mujeres que contempla la Ley 1257 de 2008 (Congreso de la República) en Colombia, "por la cual se dictan normas de sensibilización, prevención y sanción de formas de violencia y discriminación contra las mujeres [...]", como son: física, psicológica, sexual, patrimonial y económica, para posteriormente presentar algunos casos en los que se evidenciaban estos tipos de violencias e instaurar una charla con las mujeres sobre situaciones que pudieran enmarcarse en estas categorías según sus vivencias propias.

La primera mujer en intervenir relata lo que en Colombia ha sido tipificado como injuria por vía de hecho (un acto con connotaciones sexuales en espacio público) experimentado en el Sistema de Transporte Público Masivo de Bogotá -TransMilenio-. Ella se desplazaba hacia su universidad, tomó un TransMilenio que iba con la capacidad al tope, por lo que debió permanecer de pie y contra la puerta del bus, y sintió a un hombre muy cercano a ella, sin embargo, ante el aforo del bus, ella no se percata de ninguna actividad extraña hasta que una señora la aparta e insulta al hombre que iba a su lado; allí ella nota que el hombre 
estaba masturbándose y queda en shock hasta que los demás pasajeros hacen bajar al hombre del bus y lo entregan a la Policía. Cuenta que, después de estos hechos, nunca volvió a tomar un bus que la obligará a ir de pie y que prefiere esperar horas hasta que llegue un bus en el que pueda tomar asiento y sentirse más segura.

En un caso similar, otra mujer narra en la sesión que cuando tenía 11 años estaba en el parque jugando con otra niña mientras las mamás de ambas las cuidaban, en un momento ella ve a su mamá dirigirse hacia un hombre que las veía por la ventana de un edificio cercano y llamar a la Policía, ella dice que en ese momento no entendía qué pasaba, hasta que llegan los agentes y la mamá les explica que el hombre estaba masturbándose en la ventana mientras las niñas jugaban. A sus 11 años decidió (obligada) que no debía usar vestidos para ir al parque, pues podía ser víctima de situaciones similares.

Los casos de sexualización de los cuerpos y de acoso son los que más se mencionan en la sesión; otra mujer refiere que también fue víctima de violencia en el transporte público, comenta que iba en un bus camino a la oficina y que se quedó dormida en el trayecto, luego se despertó al sentir que alguien la tocaba, cuando cae en la cuenta de la situación ve a un hombre con la mano entre sus senos, quien escondía tal acción con un gabán que llevaba puesto. Ella comienza a gritar y las personas que también se transportaban en el bus le avisan al conductor, este detiene el bus y lo obliga a bajarse. Ella siente la necesidad de aclarar que no estaba usando un escote pronunciado y nos cuenta que opta por no dormirse nunca más en el transporte público para estar pendiente de quienes la rodean en los trayectos en que se moviliza.

En estos casos descritos por algunas de las mujeres que asistieron a la sesión 3, se evidencian situaciones de violencias sexuales y psicológicas que ocurren en el espacio público; las mujeres estamos expuestas a la hipersexualización de nuestros cuerpos desde muy pequeñas y el mensaje que nos entregan es el de vulnerabilidad ante actos que manifiestan deseo sin que medie consentimiento o el respeto por la autonomía sobre nuestros cuerpos.

Las mujeres somos objetivizadas para el placer masculino (MacKinnon, 1995) con los actos violentos repetitivos en el espacio público, impactando las formas en que vivimos y disfrutamos de la calle, de 
espacios de recreación, deporte y cultura, limitando nuestra movilidad, libertad y derechos para prevenir ser violentadas. La carga de la no violencia pasa a nuestras manos y nos sentimos responsables de demostrar que no fue culpa nuestra que nos violentaran, cuando son los violentos quienes deberían avergonzarse, recibir las sanciones y cambiar sus prácticas patriarcales violentas.

En esta misma sesión, otra mujer decide contar su historia de vida a partir de las violencias que vivió en la niñez y juventud. A los 15 años un hombre la drogó y abusó de ella, no obstante no pudo contarle a su mamá, pues siempre la señaló de mentirosa y de tener como objetivo destruir la familia; tampoco le creyó ni actuó al contarle que cuando iba a la casa de su tía el esposo de ella la tocaba en las piernas; entonces, ante la imposibilidad de tener respaldo y denunciar, ella generó desconfianza y miedo profundo hacia los hombres, así como inseguridades en sí misma, baja autoestima y dificultades de crear vínculos con las demás personas. Comenta que ya al ser mayor de edad, una amiga la 'vende' ${ }^{3}$ a un grupo de hombres, quienes la encierran y le dan tanto escopolamina como licor adulterado, si bien no fue violada, sí estuvo en una clínica por la ingesta de las sustancias que le obligaron a consumir.

Estas vivencias de violencia sexual, física y psicológica dejaron consecuencias de todo tipo en la mujer que relata la historia, ella menciona que su esfera profesional, laboral, social y personal se vio profundamente afectada, su miedo y rechazo a los hombres aún continúa, y la pregunta sobre el porqué del actuar masculino frente a ella o la posibilidad de tener responsabilidad ante lo ocurrido sigue latente.

En todos los casos narrados, las mujeres coinciden en el sentimiento de impotencia ante la inoperancia de la sanción para quienes cometen los actos violentos; en los casos en que relataban que se había llamado a la Policía, las mujeres mencionaban que se sentían revictimizadas ante el trato laxo que se tenía con los sujetos activos. Mencionaban que, tras un llamado de atención, los individuos quedaban en libertad y se sentían más expuestas, pues ni las autoridades ni la justicia operaban. Hay una falla transversal de las instituciones sociales, familiares y estatales 
en la respuesta de sanción y atención en estos casos; por ello, las mujeres que cuentan los casos se identifican en el sentimiento de rabia e impotencia ante la ocurrencia de violencias que con seguridad se van a repetir contra otras mujeres y seguirán en la impunidad.

\section{Identidades colectivas y resignificación de roles}

La última sesión se centró en la identificación de las características que nos hacen sentir mujeres, aquellas que desafían nuestros entornos y que a la vez logran transformar e impactar los contextos en que nos desarrollamos.

La pregunta orientadora indagaba por cuál era esa particularidad que la hacía sentir una mujer poderosa, que aportaba a su comunidad y que retaba los estereotipos; las respuestas, por supuesto, fueron tan diversas como individuos participaban.

Entre las respuestas se mencionó que ejercer una profesión como la zootecnia generaba ya una mirada distinta a las mujeres que le apostaban por este proyecto de vida; el trabajo en esa área, aunque demanda de unos esfuerzos adicionales en cuanto a horarios y dedicación, pues le ponen mayores obstáculo por ser mujer, lograba abrir camino para reafirmar su idoneidad en ese nicho profesional; otras de las mujeres se refirieron a su carácter berraco y la fuerza con que afrontaban las dificultades, resaltando que la fortaleza no es una característica que per se vaya con los roles que socialmente se asignan a las mujeres.

Finalmente, la docente habitual del grupo señala que, en la medida en que se realizan las actividades, talleres y acompañamiento en el marco del programa del CDI, se forman comunidades de apoyo entre las mujeres que permiten fortalecer el tejido social, crear una identidad de grupo y promover el uso de herramientas jurídicas y administrativas para hacer valer sus derechos en salud y como mujeres, lo que genera una agencia tanto individual como colectiva dado que identifican cómo sus propias experiencias pueden servir a otras personas del grupo y entre todas buscan estrategias para solucionar problemáticas comunes.

Se observa que durante el transcurso de las sesiones las mujeres van relatando situaciones de sexismo a través de historias de discriminación y violencia, pero también resaltan la capacidad de construirse al margen 
de la figura de víctima, mediante características como la fuerza, la resiliencia, la toma de decisiones sobre su cuerpo y el continuo proceso de repensarse - vivir el mundo desde una perspectiva crítica que se adapte a sus propias representaciones de lo femenino, para superar y desafiar los estereotipos de género-.

\section{El sexismo a la luz del derecho penal}

Los avances normativos en materia de protección, reivindicación y reconocimiento de los derechos de las mujeres en Colombia han sido tangibles durante las últimas décadas, pasamos de un Estado que reconocía la agravación punitiva de la violencia sexual entre cónyuges solamente para evitar el efecto negativo en las familias y los hijos menores (García, 2013), como lo evidenciaba la exposición de motivos de la Ley 599 de 2000, a un Estado que reconoce cinco tipos de violencias contra las mujeres a partir de la Ley 1257 de 2008, crea el tipo autónomo del feminicidio con la Ley 1761 de 2015, popularmente conocida como Rosa Elvira Cely (Congreso de la República de Colombia, 2015), e instituye una línea jurisprudencial que advierte sobre la responsabilidad del Estado en la construcción de las violencias de género, alerta de las consecuencias de la discriminación y subordinación contra la mujer, y exhorta a que todos los esfuerzos institucionales estén dirigidos a la restauración de los derechos vulnerados, la protección de las mujeres y la construcción de instituciones que garanticen la igualdad de oportunidades, no solo en materia de acceso a la justicia, sino en la totalidad de los ámbitos institucionales, sociales y económicos.

En este sentido, la jurisprudencia ha resaltado que los delitos como el feminicidio y los demás violencias contra las mujeres deben entenderse bajo una trayectoria histórica que incluya las variables estructurales que sustentan sociedades construidas sobre un sistema patriarcal; por ejemplo, en materia de feminicidio, la Corte Constitucional ha indicado: "Este tipo penal se diferencia del homicidio en las motivaciones del autor, en tanto se basa en una ideología discriminatoria fundamentada en la desvalorización de la condición humana y social de la mujer, y por 
tanto en imaginarios de superioridad y legitimación para ejercer sobre ellas actos de control, castigo y subordinación" (Sentencia C-297/16).

De igual forma, la Comisión Asesora de Política Criminal, en su informe "Diagnóstico y propuesta de lineamientos de política criminal para el Estado colombiano" (2012), destacó la necesidad de integrar el enfoque de género al sistema penal, con el fin de lograr una visión más amplia de la situación en el país que logre articular las vivencias del $51 \%$ de la población, que lo representan las mujeres, y así diseñar e implementar estrategias eficaces en la criminalización primaria, secundaria y terciaria relacionadas con los delitos que afectan principalmente a las mujeres o en donde se comprometan sus derechos mínimos, como lo es la interrupción voluntaria del embarazo, que, aunque se ha recomendado por casi una década su despenalización para garantizar los derechos reproductivos, a la vida y a la salud de las mujeres, aún no se avanza jurídicamente en tal materia: "En efecto, aunque dentro de los tratados ratificados por Colombia no existe la obligación expresa de despenalizar el aborto, lo cierto es que los estándares internacionales se orientan a la eliminación de las disposiciones legales y prácticas que impidan el pleno ejercicio de los derechos sexuales y reproductivos y generen situaciones discriminatorias. [...]".

Tanto expertos en política criminal y jueces como organizaciones de mujeres, colectivos de derechos humanos y la población en general han acompañado el proceso de visibilizar la situación de vulneración de derechos en Colombia contra las mujeres, y, en consecuencia, han brindado distintas herramientas que van desde lo académico hasta lo jurídico para hacer apuestas conjuntas de prevención, acceso a la justicia y sanción en casos relacionados con la VBG.

La prevención pasa por la deconstrucción de instituciones que se han arraigado históricamente en el derecho penal, como fue el caso de los 'crímenes pasionales', bajo la cual se justificaba el asesinato de las mujeres con el argumento del exceso de amor:

[...] el criminal pasional se entiende como si no fuera peligroso para la sociedad. Esto obedece a que se caracteriza al criminal como si actuara fuera de sí, sin mediación de la conciencia racional, poseído por una intensa emoción. De allí se deriva su no responsabilidad plena 
y, por lo tanto, su castigo atenuado. El lenguaje ordinario es elocuente cuando designa como crimen pasional aquel homicidio que acontece en razón de los vínculos amorosos y en medio de un arrebato sentimental (Jimeno, 2019, p. 87).

Si bien con posterioridad se eliminó tal categoría del uso jurisprudencial, aún queda en los imaginarios colectivos de los agentes judiciales que hay motivos menos reprochables cuando se asesina a una mujer, reforzando entonces la idea de posesión sobre el cuerpo de las mujeres y reiterando que una mujer que decide romper una relación sentimental o ser infiel afecta gravemente la honra de su cónyuge y merece tal pena.

El activismo académico de profesoras como Myriam Jimeno, para denunciar que los crímenes pasionales como categoría jurídico-penal consolidaba los estereotipos de género que ayudaban a la continuidad de las violencias contra las mujeres; de Isabel Agatón Santander, con su apuesta por la inclusión de la justicia de género como un norte necesario en la jurisprudencia colombiana para superar "los obstáculos que enfrentan las mujeres para acceder a la justicia [...] con el propósito de desvirtuar atributos propios del ámbito jurídico, como la neutralidad, la completitud y la coherencia, y de hacer visible el carácter de subordinación de las mujeres y de la dominación masculina, sustentadas en disposiciones normativas y en decisiones judiciales" (2013); de otras académicas y de muchas organizaciones feministas, de mujeres y de derechos humanos más han coadyuvado en la evolución del derecho penal para que se desdibuje su rol estático y aislado de las realidades de los sujetos a quienes se dirige y comience a advertir la necesidad de un rol más activo y consciente de su capacidad de visibilizar y prevenir violencias futuras a partir de acciones afirmativas que surjan en sus procedimientos.

No obstante el avance normativo y jurisprudencia, el país demuestra un estancamiento en las tasas de impunidad, la prevención efectiva de la VBG y, sobre todo, en la transformación cultural para concertar entornos libres de sexismo, lo que deriva en el aumento constante de violencias contra las mujeres.

Para el año 2020 con corte a octubre, el país registraba 359 casos de feminicidios (Gallo, 2020); entre enero y octubre de 2019, el Instituto 
Nacional de Medicina Legal y Ciencias Forenses reportaba 98583 casos de violencia de género, para ese período las cifras se distribuían así: "Por violencia de pareja 34183 casos; por violencia interpersonal 31044 agresiones; por violencia sexual 18967; por violencia intrafamiliar 13160 casos" (García, 2019).

Para el año 2020, una mujer fue víctima de violencia sexual cada 34 minutos; en los casos de violencia por parte de su pareja o expareja, ocurrió un hecho contra una mujer cada 19 minutos; y se perpetró un feminicidio íntimo cada 3 días (Sisma Mujer, 2020).

Estas cifras no reflejan la realidad de los casos de VBG en Colombia, pues es claro que hay un subregistro ante todos los casos que no llegarán al conocimiento de una autoridad; las mujeres tienen miedo de denunciar cuando conviven con su pareja, hay obstáculos para que accedan a la atención social y psicológica que se necesita para identificar un ciclo de violencias, combatirlo, denunciarlo y cerrarlo.

Las mujeres siguen siendo asesinadas como consecuencia de una sociedad patriarcal que reproduce y actualiza en el día a día los postulados que determinan que deben ser tratadas como propiedad, objetivizadas, hipersexualizadas y violentadas.

El ordenamiento jurídico penal colombiano evidencia insuficiencia a la hora de tramitar todos los casos que son de su conocimiento: “De acuerdo con la Fiscalía General de la Nación, de enero de 2017 a septiembre de 2019, de 115000 casos de delitos sexuales en contra de mujeres, 99000 están en etapa de indagación. [...] Es decir, que no se ha hecho ni siquiera la primera audiencia" (Gutiérrez, 2019).

Por una parte, encontramos la brecha en el acceso a la justicia que enfrentan las mujeres al tener que acudir a un sistema ineficiente y tardío, en donde saben que, a pesar de denunciar, van a tener obstáculos por las resistencias que pondrán los operadores judiciales al interponer sus estereotipos antes que la función pública que desarrollan (Equidad de la Mujer, Presidencia de la República de Colombia, 2015), la congestión judicial y la dificultad probatoria que existe en los casos de violencia de género al requerir pruebas contextuales; y, por otro, el abandono estatal en las fases previas a las etapas de violencia basada en género, como en los casos de feminicidio, que en el ciclo de violencias anterior al asesinato habitualmente incluye delitos como lesiones o violencia 
intrafamiliar, que son de interés del derecho penal pero que en su trámite se tratan de forma aislada de la violencia basada en género, sin advertir las consecuencias fatales en que puede derivar para las mujeres.

\section{Futuro del derecho penal para abordar un enfoque de género}

En la pirámide de los actos de odio, se identifican varias etapas en las que el ordenamiento jurídico no intervendrá y que, sin embargo, están gestando actos posteriores de violencia que pueden tener resultados mortales. La pirámide está constituida así:

Figura 1. Pirámide de actos de odio

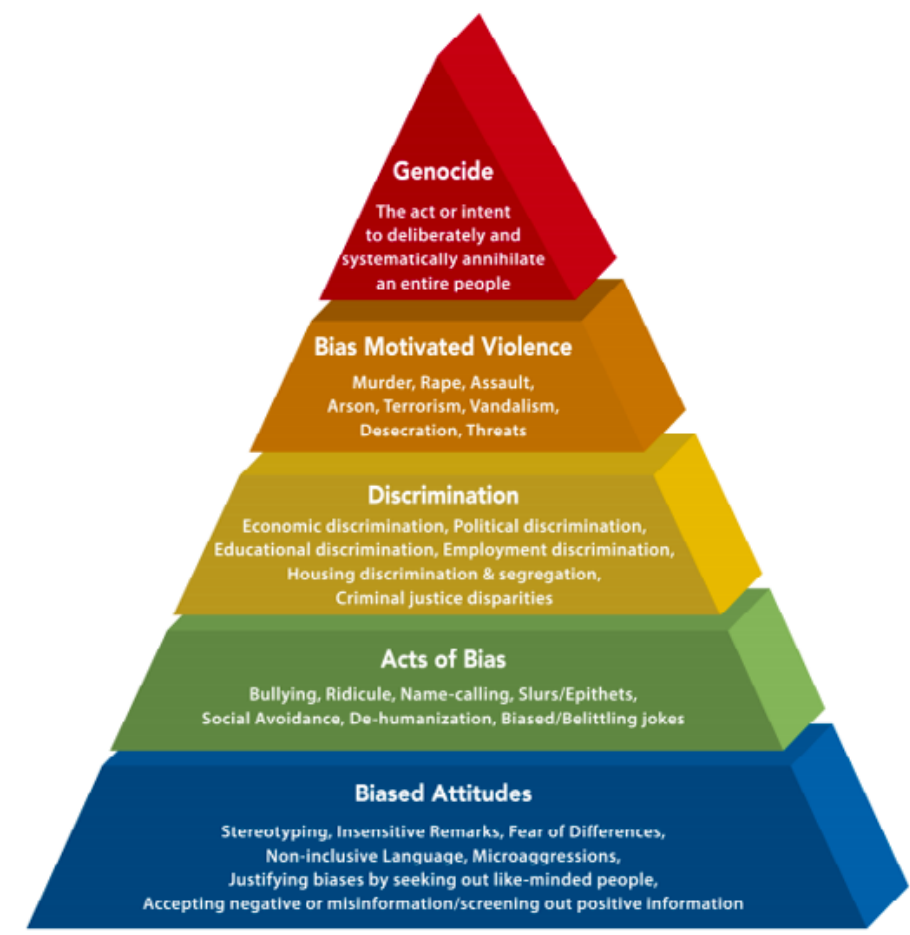

Fuente: https://www.adl.org/sites/default/files/documents/pyramid-of-hate.pdf

La pirámide muestra los diferentes tipos de odio que ha clasificado la psicología: en la base están los actos estereotipados, omitir el lenguaje 
inclusivo, aceptar información no verificada; luego, está el matoneo, la deshumanización del otro y el asignar etiquetas, 'apodos'; le sigue la discriminación institucional en acceso a empleo, disparidad en el sistema judicial; más arriba, están los actos motivados por los sesgos o prejuicios, como los crímenes de odio; y en la punta, está el delito máximo que puede cometerse por odio, el genocidio (destrucción o la tentativa de destrucción intencional, en todo o en parte, de un grupo nacional, étnico, racial o religioso).

Para Sally Kohn (2018), las divisiones que ilustra la pirámide están ligadas una con otra y lo que demuestran es la evolución repetitiva que tienen los mensajes de odio; por supuesto que no todos derivan en genocidios o feminicidios para el caso de VBG sobre el que hemos trabajado a lo largo de este texto, pero todos los casos de feminicidios y genocidios sí tienen una trayectoria que recoge las etapas previas que se evidencian en la figura 1.

Por ello, a pesar de que el derecho penal solo aborda los daños efectivos a bienes jurídicos protegidos y que los actos que serán de su interés deben obedecer al principio de legalidad y estar tipificados, es necesario que desde la política criminal sí se priorice la prevención en materia de VBG en las etapas más tempranas de la construcción del sexismo, pues es allí donde se continuarán situando los imaginarios colectivos que justifican la discriminación y violencias contra las mujeres.

Con los relatos de las mujeres de Usaquén se hace visible la ocurrencia rutinaria de actos sexistas que atraviesan la vida de personas con experiencias, profesiones, familias e historias distintas; sin importar el contexto en el que se crezca, cada mujer tiene un relato que contar en el que los estereotipos de género han marcado su vida, han limitado la forma en que hace uso del espacio público, han determinado qué deportes puede practicar, han destruido su autoestima, han generado una ruptura en la confianza con las instituciones que deberían protegerlas, han puesto en riesgo su salud y han intervenido en la construcción de su identidad como mujer individual y colectiva.

Si bien la mayoría de las situaciones narradas por las mujeres con las que se desarrollaron las sesiones no están enmarcadas dentro de una esfera de competencia propia del derecho penal, sí se puede inferir que algunas violencias posteriores pueden ser el resultado de la omisión 
estatal de tener una respuesta precisa a estos hechos iniciales. Por otra parte, urge la intervención en materia de políticas urbanísticas, culturales, educativas y sociales que transformen estos escenarios que ya son comunes en las interacciones dentro del espacio público, que repiten actos de acoso y violencias, sin tener ningún registro, intervención, ni acompañamiento para las mujeres que se ven sometidas a tales situaciones.

La responsabilidad del Estado y el llamado a la acción es lo que se busca con cada pauta normativa que se ha logrado a partir de instrumentos internacionales, como la Convención sobre la Eliminación de todas las Formas de Discriminación contra la Mujer (Cedaw) (Naciones Unidas, 1979), en donde se establece: "Artículo 3․ Los Estados partes tomarán en todas las esferas, y en particular en las esferas política, social, económica y cultural, todas las medidas apropiadas, incluso de carácter legislativo, para asegurar el pleno desarrollo y adelanto de la mujer, con el objeto de garantizarle el ejercicio y el goce de los derechos humanos y las libertades fundamentales en igualdad de condiciones con el hombre" (Naciones Unidas, 1979).

La Convención de Belem do Pará (Organización de los Estados Americanos, 1994), que a su vez indica:

Artículo $4^{\circ}$. Toda mujer tiene derecho al reconocimiento, goce, ejercicio y protección de todos los derechos humanos y a las libertades consagradas por los instrumentos regionales e internacionales sobre derechos humanos. Estos derechos comprenden, entre otros:

a) el derecho a que se respete su vida; b) el derecho a que se respete su integridad física, psíquica y moral; c) el derecho a la libertad y a la seguridad personales; d) el derecho a no ser sometida a torturas; e) el derecho a que se respete la dignidad inherente a su persona y que se proteja a su familia; f) el derecho a igualdad de protección ante la ley y de la ley; g) el derecho a un recurso sencillo y rápido ante los tribunales competentes, que la ampare contra actos que violen sus derechos; h) el derecho a libertad de asociación; i) el derecho a la libertad de profesar la religión y las creencias propias dentro de la ley, y j) el derecho a tener igualdad de acceso a las funciones públicas de 
su país y a participar en los asuntos públicos, incluyendo la toma de decisiones (Organización de los Estados Americanos, 1994).

Y la Declaración y Plataforma de Acción de Beijing (Naciones Unidas, 1995), en donde los Estados se comprometen a:

[...] crear condiciones necesarias para la potenciación del papel de la mujer en la sociedad. Tiene por objeto acelerar la aplicación de las Estrategias de Nairobi orientadas hacia el futuro para el adelanto de la mujer y eliminar todos los obstáculos que dificultan la participación activa de la mujer en todas las esferas de la vida pública y privada mediante una participación plena y en pie de igualdad en el proceso de adopción de decisiones en las esferas económica, social, cultural y política. Esto también supone el establecimiento del principio de que mujeres y hombres deben compartir el poder y las responsabilidades en el hogar, en el lugar de trabajo y, a nivel más amplio, en la comunidad nacional e internacional. La igualdad entre mujeres y hombres es una cuestión de derechos humanos y constituye una condición para el logro de la justicia social, además de ser un requisito previo necesario y fundamental para la igualdad, el desarrollo y la paz. Para obtener el desarrollo sostenible basado en el ser humano, es indispensable que haya una relación transformada, basada en la igualdad, entre mujeres y hombres. Se necesita un empeño sostenido y a largo plazo para que mujeres y hombres puedan trabajar de consuno para que ellos mismos, sus hijos y la sociedad estén en condiciones de enfrentar los desafíos del siglo XXI (Naciones Unidas, 1995, p. 18).

Estos compromisos globales instan a reconocer, actuar y promover intervenciones integrales para que las condiciones de las mujeres mejoren en todas las esferas, desde la autonomía económica hasta en su participación política e incidencia en temas de paz, a la vez que conduce a los Estados a evaluar sus niveles de responsabilidad en la obstrucción del acceso a estos derechos básicos y la inoperancia ante situaciones sociales en las que ocurren actos de violencia contra las mujeres.

En consecuencia, el Estado colombiano, con la ratificación de los convenios internacionales y la expedición de las leyes internas correspondientes a la prevención, eliminación de todas las formas de violencias 
contra las mujeres y la creación de oportunidades para promover la garantía plena de los derechos, no solamente debe procurar la atención institucional al momento de recibir reportes por casos de discriminación o cualquier tipo de violencias basadas en género, sino que debe generar una ruta de acción transversal a todos los servicios que prestan las instituciones en materia de educación, salud, empleo, cultura ciudadana y todos los demás que estén bajo el ámbito estatal, para que desde las etapas más tempranas se consoliden prácticas sociales que estén libres de sexismo.

Para cumplir tales objetivos, es indispensable que se edifique desde los territorios, conociendo las dinámicas locales, aprovechando las enseñanzas y formas de organización social que ya han logrado las 8 mujeres desde las esferas más inmediatas. El tejido social que entre todas las mujeres han construido debe ser la base de las políticas de prevención de las violencias basadas en género. Es la agencia colectiva como mujeres la que permitirá gestar, cuidar y estructurar las estrategias de escucha, transformación cultural y resignificación de las relaciones de poder, habitualmente encaminadas a imponer la subordinación del otro, pero que, desde la perspectiva de los procesos territorializados de las mujeres, pueden convertirse en rupturas reales y sólidas a las dinámicas patriarcales.

El Estado debe entonces aprovechar lo que ya han obtenido las mujeres en redes de apoyo y defensa conjunta de los derechos, a la vez que promover espacios que permitan este tipo de configuraciones entre las personas. Como lo recomendó el informe de política criminal, se debe utilizar el enfoque de género en cada etapa y proceso que se disponga para que las voces de las mujeres queden también plasmadas en las decisiones institucionales y en los modelos de criminalización primaria, secundaria y terciaria.

Finalmente, hay que hacer un llamado a la sororidad, que incluya a las mujeres en sus territorios y a quienes representen cada una de las instancias de toma de decisiones relacionadas con el diseño e implementación de la política criminal, para que se promuevan a su vez espacios e iniciativas sociales que reevalúen las formas en que usamos el discurso para dividir y procuremos un discurso tanto popular como 
jurídico, que, como lo propuso Bell Hooks en "Sisterhood: political solidarity between women" (1986), logre unirnos por los intereses y creencias compartidas, en la valoración y exaltación de la diversidad, en nuestras batallas para terminar las relaciones de opresión y en políticas de la solidaridad.

\section{Conclusiones}

El presente artículo hace un recorrido por algunos momentos de las historias de vida de las mujeres que participaron en las sesiones de discusión sobre el sexismo, en sus manifestaciones a través de las violencias de género y la discriminación, el discurso que construye realidades y las implicaciones que ha generado en cada una las construcciones de identidad estereotipadas.

No solamente se busca poner sobre la mesa las situaciones rutinarias que afrontan las mujeres y las secuelas a largo plazo tanto en el ámbito personal como en el público y en su relacionamiento incluso con las instituciones y autoridades que deja el sexismo en sus múltiples formas de manifestación, sino que se registró la trayectoria que cursa la violencia desde sus formas micro hasta aquellas que ya están tipificadas y requieren de la intervención del derecho penal.

El artículo permite a quien lo lee identificar unas herramientas de análisis donde el discurso guía la ruta para distinguir los antecedentes de la violencia y quizá posteriormente incluir tal estrategia a fin de ampliar la posibilidad de detectar los factores de riesgo que debe tener en cuenta el derecho penal para prevenir que las violencias contra las mujeres terminen en actos de feminicidio.

Por último, el texto establece una serie de propuestas para que se incluyan variables que permitan eliminar los obstáculos en el acceso a la justicia que se ponen a las mujeres y trazar un giro en el derecho penal que ayude a construir desde un enfoque de género, que no solo se deberá implementar en materia procesal, sino también en el diseño de la política criminal. 


\section{Referencias}

\section{Libros y capítulos de libros}

Adichie, C. (2018). Querida Ijeawele. Cómo educar en el feminismo. Bogotá: Penguin Random House.

Agatón, I. (2013). Justicia de género, un asunto necesario. Bogotá: Temis.

Austin, J. (2014). Cómo hacer cosas con palabras. Barcelona: Ediciones Paidós. Butler, J. (2004). Lenguaje, poder e identidad. Madrid: Editorial Síntesis S.A.

Foucault, M. (1992). El orden del discurso. Buenos Aires: Letra E.

Foucault, M. (2010). El coraje de la verdad. Buenos Aires: Fondo de Cultura Económica.

Jimeno, M. (2019). Cultura y violencia: hacia una ética social del reconocimiento. Bogotá: Universidad Nacional de Colombia.

Kohn, S. (2018). The opposite of hate: a field guide to repairing our humanity. New York: Algonquin Books of Chapel Hill.

Lagarde, M. (1996). Identidad de género y derechos humanos. Construcción de las humanas. En Estudios básicos de derechos humanos. Tomo IV (pp. 6394). San José, Costa Rica: Instituto Interamericano de Derechos Humanos. MacKinnon, C. (1995). Hacia una teoría feminista del Estado. Madrid: Ediciones Cátedra S.A.

Sau, V. (2000). Diccionario ideológico feminista I. Barcelona: Icaria-La Mirada Esférica.

Tsesis, A. (2002). Destructive messages how hate speech paves the way for harmful social movements. New York: New York University Press.

\section{Artículos}

García, L. (enero-junio, 2013). El contexto de mujer en la realidad jurídicopenal colombiana: delitos sexuales y revictimización. IUSTA, (38), 103-131.

Hooks, B. (1986). Sisterhood: political solidarity between women. Feminist Review, 23(1), 125-138.

\section{Normatividad nacional e internacional}

Colombia, Congreso de la República. Ley 599 de 2000 (24 de julio). Código Penal Colombiano. 
Colombia, Congreso de la República. Ley 1257 de 2008 (4 de diciembre).

Colombia, Congreso de la República. Ley Rosa Elvira Cely, Ley 1761 de 2015 (6 de julio).

Naciones Unidas. (1979). Convención sobre la Eliminación de todas las Formas de Discriminación contra la Mujer. Recuperado de https://www.ohchr. org/sp/professionalinterest/pages/cedaw.aspx

Naciones Unidas. (1995). Declaración y Plataforma de Acción de Beijing.

Organización de los Estados Americanos. (1994). Convención Interamericana para Prevenir, Sancionar y Erradicar la Violencia contra la Mujer "Convención de Belém do Pará". Recuperado de https://www.oas.org/juridico/ spanish/tratados/a-61.html

\section{Sentencias}

Colombia, Corte Constitucional. Sentencia C-297 de 2016, Expediente D-11027 (8 de junio).

Corte Interamericana de Derechos Humanos. Veliz Franco y otros versus Guatemala (19 de mayo de 2014).

\section{Informes}

Comisión Asesora de Política Criminal. (2012). Informe final. Diagnóstico y propuesta de lineamientos de política criminal para el Estado colombiano. Bogotá: Ministerio de Justicia y del Derecho.

Equidad de la Mujer, Presidencia de la República de Colombia. (2015). Segunda medición del estudio sobre tolerancia social e institucional de las violencias contra las mujeres. Bogotá: Presidencia de la República de Colombia.

Sisma Mujer. (2020). La pandemia antes del Covid: violencias hacia las mujeres y niñas durante 2019 y 2020 en Colombia. Bogotá: Sisma Mujer.

\section{Entrevistas}

Gallo, G. (13 de octubre de 2020). ¿Cuáles son las cifras sobre la violencia contra las mujeres en Colombia? (R. N. Colombia, entrevistador).

García, C. (27 de noviembre de 2019). Medicina Legal advierte que este año han sido violentadas más de 98 mil mujeres. (J. R. Angarita, entrevistador). 
Gutiérrez, H. (3 de diciembre de 2019). La violencia de género y su 'normalización' en Colombia. RCN Radio. Recuperado de https://www.rcnradio. com/recomendado-del-editor/la-violencia-de-genero-y-su-normalizacionen-colombia

\section{Páginas web}

ICBF. (20 de noviembre de 2020). Centro de Desarrollo Infantil. Recuperado de https://www.icbf.gov.co/portafolio-de-servicios-icbf/centro-de-desarrolloinfantil 